\title{
Measurement of Inflammatory Cytokines and Thrombomodulin in Chronic Subdural Hematoma
}

\author{
Masatoshi KITAZONO, ${ }^{1}$ Hiroyuki YOKOTA, ${ }^{1}$ Hidetaka SATOH, ${ }^{1}$ Hidetaka ONDA, ${ }^{1}$ \\ Gaku MATsumoto, ${ }^{1}$ Akira Fuse, ${ }^{1}$ and Akira TERAMOTO ${ }^{2}$
}

Departments of ${ }^{1}$ Emergency and Critical Care Medicine and

${ }^{2}$ Neurosurgery, Nippon Medical School, Tokyo

\begin{abstract}
Inflammation and the coagulation system may influence the genesis of chronic subdural hematoma (CSDH). The appearance of CSDH on computed tomography (CT) varies with the stage of the hematoma. This study investigated the pathogenesis and the recurrence of CSDH by comparing cytokine levels with the CT features of CSDH in 26 patients with 34 CSDHs who underwent single burr-hole surgery at our hospital between October 2004 and November 2006. The hematoma components removed during the procedure were examined, and the hematoma serum levels of cytokines measured such as thrombomodulin (TM), interleukin-6 (IL-6), tumor necrosis factor- $\alpha$ (TNF $\alpha$ ), and interleukin-10 (IL-10). Using CT, mixed density hematomas were distinguished from other homogeneous hematomas, and found that the TM level was significantly higher in mixed density hematomas than in homogeneous hematomas (p $=0.043$ ). Mixed density hematomas were classified into three subtypes (laminar, separated, and trabecular hematomas). The TM level was significantly higher in laminar and separated hematomas than in other hematomas ( $p=0.01$ ). The levels of IL-6, TNF $\alpha$, and IL-10 were extremely high, but showed no significant differences in relation to the CT features. Mixed density hematomas had high recurrence rate, as reported previously, and TM level was high in mixed density hematomas such as laminar and separated mixed density hematomas. The present findings suggest that the types of CSDH associated with high TM levels tend to have higher recurrence rate.
\end{abstract}

Key words: chronic subdural hematoma, computed tomography, cytokines, thrombomodulin, interleukin

\section{Introduction}

Chronic subdural hematoma (CSDH) commonly requires neurosurgical treatment, and often recurs after surgery. Previous studies have suggested that local anticoagulation and inflammatory changes may influence the pathogenesis of CSDH, although the process has not been clearly elucidated. ${ }^{16,28,32,35,38)}$ The transformation of traumatic subdural hygromas into CSDH has been discussed in several studies. ${ }^{7,23)}$

Computed tomography (CT) of CSDH often shows changes in the components of hematomas, and there may be an association between CT appearance and altered cytokine levels in CSDH. ${ }^{15,19,20)}$ In particular, thrombomodulin (TM) has an anticoagulatory function and is expressed after endothelial cell injury. ${ }^{5,10)}$
TM has been measured in hematomas and has been implicated in the expansion of CSDH. ${ }^{18)}$ However, no studies have evaluated the relationship between TM in CSDH and the CT characteristics. Many studies have found that the recurrence rate of CSDH is correlated with the CT findings.

The present study measured the TM levels in $\mathrm{CSDH}$ with various CT findings, and investigated the relationship between TM level in CSDH and recurrence of $\mathrm{CSDH}$. The inflammatory cytokines interleukin-6 (IL-6) and tumor necrosis factor- $\alpha$ $(\mathrm{TNF} \alpha)$, as well as the anti-inflammatory cytokine interleukin-10 (IL-10) were also measured. The CT appearance of the lesions were also evaluated.

Received October 31, 2011; Accepted January 20, 2012

Author's present address: Masatoshi Kitazono, MD, Department of Emergency and Critical Care Center, Kawaguchi Municipal Medical Center, Kawaguchi, Saitama, Japan. 


\section{Materials and Methods}

This study included 26 patients, 22 men and 4 women aged 32-98 years (mean 73.8 years), with 34 CSDHs treated in our hospital from October 2004 to November 2006. Six patients had bilateral hematomas and two patients had recurrence of hematoma. The mean Glasgow Coma Scale score of patients on admission was 13.5 (range 3-15). No patient had coagulopathy or systemic inflammatory changes detected by blood testing or in their past medical history. The diagnosis of CSDH was established using CT in all cases. The CT appearance of hematomas was evaluated according to density and internal architecture. Hematomas were classified into low density (6 hematomas), iso density (10 hematomas), and mixed density (18 hematomas). No patient had a high density hematoma. Additionally, low, iso, and high density hematomas were characterized as the homogeneous type, and mixed density hematomas were classified as laminar (5 hematomas), separated (9 hematomas), or trabecular types (4 hematomas). Mixed density hematomas were subdivided because of the changes in appearance by the point of development. ${ }^{19,20)}$ Laminar type hematomas had a thin high density layer along the inner membrane, separated type hematomas contained two components of



Fig. 1 Computed tomography scans demonstrating examples of three types of mixed density chronic subdural hematoma: laminar (A), separated (B), and trabecular (C) types. different densities with a clear boundary, and trabecular type hematomas had a high density septum running between the inner and outer membranes on a mixed density background. Figure 1 demonstrates examples of these CT subtypes. The CT classification of hematomas was based on discussion in our institution by three or more qualified neurosurgeons, certified by the Japan Neurosurgical Society. After diagnosis, single burr-hole drainage of the hematoma was performed. The hematoma serum removed during this procedure was separated by centrifugation at $3000 \mathrm{~g}$ for 10 minutes and stored at $-70^{\circ} \mathrm{C}$ until analysis.

Enzyme immuno assay was used to measure TM (normal range 2.1-4.1 FU/ml in male, 1.8-3.9 FU/ml in female), chemiluminescent enzyme immunoassay to measure IL-6 (normal range $\leq 4.0 \mathrm{pg} / \mathrm{ml}$ ), and enzyme-linked immunosorbent assay to measure IL-10 (normal range $\leq 5 \mathrm{pg} / \mathrm{ml}$ ) and $\mathrm{TNF} \alpha$ (normal range $0.6-2.8 \mathrm{pg} / \mathrm{ml}$ ). The values of IL-6 and IL-10 were expressed as the median, interquartile range, and range. The values of $\mathrm{TM}$ and $\mathrm{TNF} \alpha$ were expressed as the mean \pm standard deviation (SD), and the Mann-Whitney test was used to compare the various stages of hematoma. Statistical analysis was performed using commercially available statistical software (SPSS 16.0.2; IBM, Armonk, New York, USA).

This study was conducted in accordance with the ethical standards of the Nippon Medical School Hospital ethics committee. All participants provided written informed consent.

\section{Results}

Table 1 provides an overview of patient characteristics (age, sex, clinical symptoms on admission, midline shift on CT, and postoperative recurrence). Neither age nor sex affected the values of any cytokines. The levels of TM, IL-6, IL-10, and TNF $\alpha$ in hematomas were higher than normal values; in

Table 1 Overview of patient characteristics

Low density hematoma $(n=6)$ Iso density hematoma $(n=10)$ Mixed density hematoma $(n=18)$

\begin{tabular}{lccc}
\hline Mean age (range), yrs & $73.83(56-92)$ & $70.8(32-92)$ & $75.44(33-98)$ \\
Sex (male/female) & $5 / 1$ & $9 / 1$ & $15 / 3$ \\
Mean GCS score (range) & $12.17(3-15)$ & $14.3(12-15)$ & $13.44(8-15)$ \\
Paresis & 3 & 4 & 14 \\
Aphasia or dysarthria & 1 & 3 & 3 \\
Midline shift & 4 & 5 & 9 \\
Recurrence & 1 & 0 & 1
\end{tabular}

GCS: Glasgow Coma Scale. 
particular, the levels of IL-6, IL-10, and TNF $\alpha$ were markedly higher (Tables 2 and 3). The values of IL-6, IL-10, and TNF $\alpha$ showed no significant differences with CT density and appearance. The level of TM was significantly higher in mixed density hematomas than in homogeneous hematomas $(p<0.05)$ (Fig. 2). The average TM level was $4.9 \pm 2.0 \mathrm{FU} / \mathrm{ml}$ (mean $\pm \mathrm{SD}$ ) in mixed density hematomas $(\mathrm{n}=18)$ and $4.3 \pm 3.3 \mathrm{FU} / \mathrm{ml}$ in homogeneous hematomas $(\mathrm{n}=16)(\mathrm{p}=0.043)$.

The value of $\mathrm{TM}$ was $6.2 \pm 2.0 \mathrm{FU} / \mathrm{ml}$ in laminar hematomas ( $\mathrm{n}=5$ ), and $3.5 \pm 0.8 \mathrm{FU} / \mathrm{ml}$ in trabecular hematomas $(n=4)(p=0.026)$. No differences were found between other subtypes of mixed density hematomas (laminar, separated, and trabecular types). Laminar and separated hematomas may have similarly high recurrence rates, ${ }^{19)}$ and we found significantly higher TM values in laminar and separated hematomas compared with other types $(p<0.05)$ (Fig. 3). The TM value was $5.3 \pm 2.0 \mathrm{FU} / \mathrm{ml}$ in laminar and separated hematomas $(\mathrm{n}=14)$ and $4.2 \pm$ $2.9 \mathrm{FU} / \mathrm{ml}$ in homogeneous and trabecular hematomas $(n=20)(p=0.01)$.

\section{Discussion}

CSDH shows changes in CT density with time. The initial stage appears as low density on CT, the mature stage as iso or high density, the progressive stage as mixed density, and the resolving stage as

Table 2 Values of thrombomodulin (TM) and tumor necrosis factor- $\alpha$ (TNF $\alpha)$ in chronic subdural hematomas

\begin{tabular}{lcc}
\hline \multicolumn{1}{c}{ Hematoma } & $\mathrm{TM}(\mathrm{FU} / \mathrm{ml})$ & $\mathrm{TNF} \alpha(\mathrm{pg} / \mathrm{ml})$ \\
\hline Low density $(\mathrm{n}=6)$ & $4.7 \pm 4.4$ & $8910.0 \pm 7708.1$ \\
Iso density $(\mathrm{n}=10)$ & $4.1 \pm 2.7$ & $8763.0 \pm 4810.0$ \\
Mixed density $(\mathrm{n}=18)$ & $4.9 \pm 2.0$ & $8136.1 \pm 4113.0$ \\
\hline
\end{tabular}

Values are means \pm standard deviation. Normal range of $\mathrm{TM}$ (enzyme immuno assay): 2.1-4.1 $\mathrm{FU} / \mathrm{ml}$ in male, 1.8-3.9 FU/ml in female. Normal range of TNF $\alpha$ (enzymelinked immunosorbent assay): $0.6-2.8 \mathrm{pg} / \mathrm{ml}$.

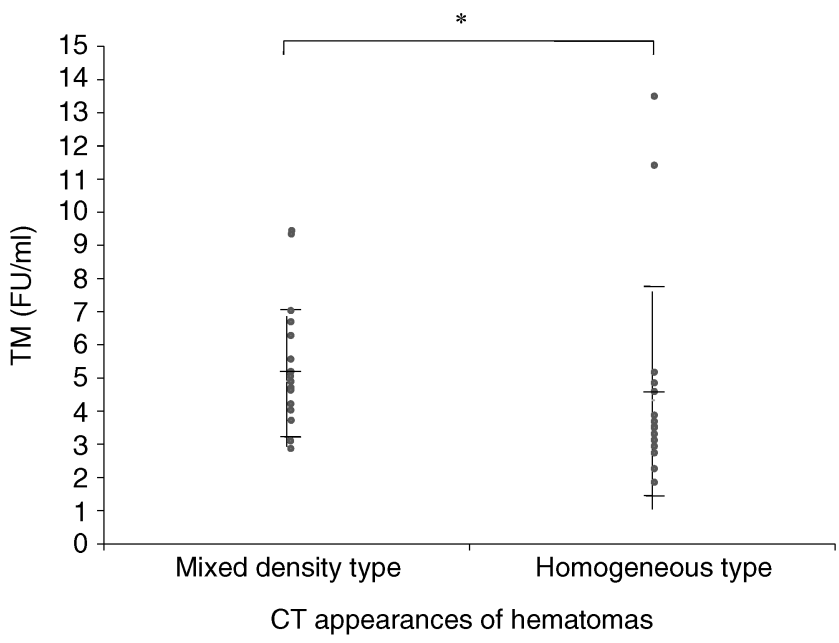

Fig. 2 Thrombomodulin (TM) level (mean \pm standard deviation) in mixed density hematomas and homogeneous hematomas. ${ }^{*} p<0.05$. CT: computed tomography.

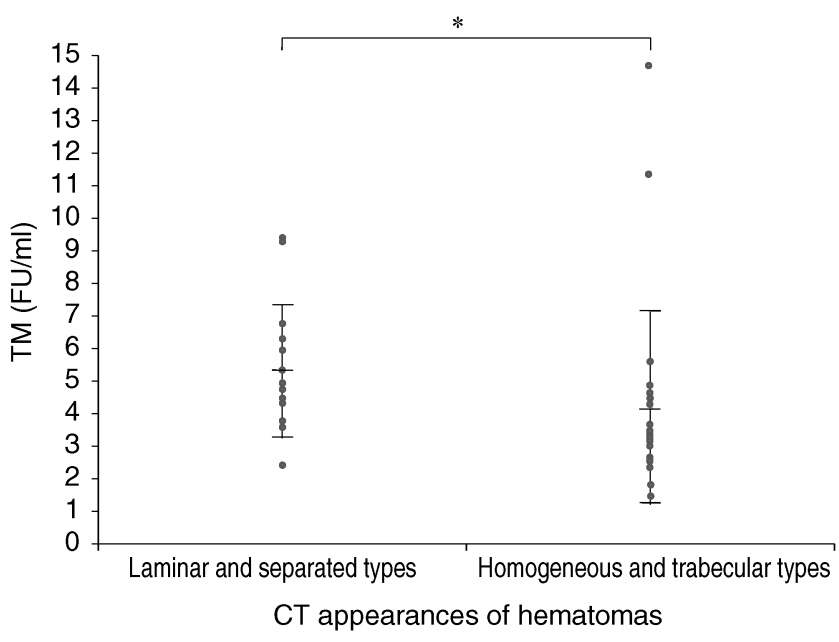

Fig. 3 Thrombomodulin (TM) level (mean \pm standard deviation) in laminar and separated hematomas versus homogeneous and trabecular hematomas. ${ }^{*} p<0.05$. CT: computed tomography.

Table 3 Values of interleukin-6 (IL-6) and interleukin-10 (IL-10) in chronic subdural hematomas

\begin{tabular}{lll}
\hline Hematoma & \multicolumn{1}{c}{ IL-6 $(\mathrm{pg} / \mathrm{ml})$} & \multicolumn{1}{c}{ IL-10 $(\mathrm{pg} / \mathrm{ml})$} \\
\hline Low density & $17900 / 6060-22525 / 3210-96900$ & $22 / 3.75-38.75 / 2-271$ \\
Iso density & $10100 / 5535-19600 / 2080-78200$ & $13 / 5.5-60 / 2-120$ \\
Mixed density & $12450 / 7835-28650 / 1310-104000$ & $28.5 / 8.75-81.75 / 2-217$ \\
\hline
\end{tabular}

Values are median/interquartile range/range. Normal range of IL-6 (chemiluminescent enzyme immunoassay): $\leq 4.0$ $\mathrm{pg} / \mathrm{ml}$. Normal range of IL-10 (enzyme-linked immunosorbent assay): $\leq 5.0 \mathrm{pg} / \mathrm{ml}$. 
relatively low density or low density without mass sign. ${ }^{15,30)}$ Some studies have grouped low, iso, and high density hematomas as a homogeneous stage, whereas the laminar stage may be a subtype of the homogeneous stage. ${ }^{19)}$ However, this study classified laminar hematomas as a subtype of mixed density hematomas, as classification of CSDH on CT has varied between studies. ${ }^{20,30)}$

CSDH was originally described as an inflammatory disease, using the term "pachymeningitis hemorrhagica interna," ${ }^{36)}$ but the causative factor of CSDH is now known to be trauma, not inflammation. ${ }^{33)}$ Recently, some studies have highlighted the relationship between local inflammation in CSDH and the mechanism of initiation and propagation of CSDH. ${ }^{2,4)}$ Subdural fluid from patients with CSDH contained a significantly higher level of $\mathrm{TNF} \alpha$ and IL-6 than that found in blood, so that the cytokines in the subdural fluid may not permeate from the blood but are synthesized and released in situ from cells facing the subdural space or from inflammatory cells infiltrating into the space, where local and specific inflammation occurred. ${ }^{29)}$ IL-6 is a pleiotropic cytokine that influences immune and inflammatory responses and is one of the major physiological mediators of the acute phase reaction. ${ }^{14,24)}$ IL-6 can cause enlargement of the gap junction between endothelial cells and can increase vascular permeability. ${ }^{29,39)}$ We found high levels of IL- 6 and $\mathrm{TNF} \alpha$, which are inflammatory cytokines, in hematomas. However, we did not find any significant differences between the various IL-6 values in hematomas classified by CT appearance, nor between the $\mathrm{TNF} \alpha$ values.

The present study found that the level of IL-10, another anti-inflammatory cytokine, was higher than normal but did not vary significantly according to the CT density or appearance of hematomas. The levels of IL-6 and IL-10 were measured in the subdural fluid obtained from patients with CSDH, and found that patients in the low IL-10 group tended to have separated hematomas on CT, suggesting that IL-10 acts as a deactivator of inflammation in the pathogenesis of CSDH. ${ }^{37)}$ The values of IL-6, TNF $\alpha$, and IL-10 found in our data were far higher than those obtained by several previous studies. In our study, the value of IL-6 was $2549.0 \pm 395.9 \mathrm{pg} / \mathrm{ml}$ or $1321.0 \pm 258.8 \mathrm{pg} / \mathrm{dl}$; the value of $\mathrm{TNF} \alpha$ was $41.1 \pm$ $8.8 \mathrm{pg} / \mathrm{ml}$; and the value of IL-10 was $56.4 \pm 96.4$ $\mathrm{pg} / \mathrm{dl} .^{8,11,29,37)}$ Our study design did not allow us to determine the role of inflammatory change in hematomas, but the markedly elevated levels of inflammatory and anti-inflammatory cytokines in hematomas were particularly interesting findings. Our patients did not have inflammatory basic diseases or im- munological disorders, so the reasons for the markedly elevated levels of cytokines were unclear.

TM is a thrombin receptor on capillary endothelial cells that inhibits blood clotting by forming a complex with thrombin and activated protein C. ${ }^{5,6,21)}$ Vascular endothelial injury increases the plasmasoluble TM level. ${ }^{10,31)}$ The plasma-soluble TM level was higher in hematomas than in peripheral blood ( $p$ $<0.0001$ ), suggesting that the sinusoidal vessels in the outer membrane of CSDH were continuously damaged and TM was expressed, which triggered hemorrhaging from the outer membrane. ${ }^{18)} \mathrm{TM}$ inhibits hemostatic thrombus formation in the hematoma, so the CSDH continued to expand without coagulation. TM levels in hematomas were compared with the levels in peripheral blood, but the relationship between TM values and CT findings in hematomas was not evaluated. ${ }^{18)}$ In this study, we compared TM values in hematomas with CT density and appearance of the hematomas.

Recurrence after surgical treatment is common and several studies have reported average rates of recurrence of $5-30 \% .{ }^{1,3,26)}$ In an attempt to reduce the recurrence rate, various surgical methods have been used with different number of burr holes, duration of drainage, irrigation technique, and several other factors. ${ }^{40)}$

In the present study, we performed single burr hole surgery, and removed and irrigated the hematoma, but because only two patients had recurrence (1 low density hematoma, 1 laminar type hematoma) during this investigation period, we could not analyze recurrence rates. The low density hematoma recurred as trabecular type hematoma, and the laminar type hematoma recurred as iso density hematoma. We classified hematomas into three density subtypes and measured the TM values. The recurrence rate of hematoma types have been reported as homogeneous $15 \%$, laminar $19 \%$, separated $36 \%$, and trabecular $0 \% .{ }^{19)}$ In homogeneous hematomas, differences in CT density did not affect the recurrence rate. ${ }^{34)}$ Mixed density hematomas had a high recurrence rate, and the recurrence rate of the separated type was highest and that of the trabecular type was lowest.9,12,19,20,25,29) Hyperfibrinolysis might be involved, as the fibrinogen and d-dimer levels in heterogeneous hematomas were higher than those in homogeneous hematomas. ${ }^{22)}$

The present study found that TM values in separated and laminar hematomas were significantly higher than in homogeneous and trabecular hematomas, and the recurrence rate of separated and laminar hematomas is known to be significantly higher than that of other hematomas. ${ }^{19)}$ Therefore, we suggest that hematomas with high TM values tend to 
present as mixed density hematomas (particularly laminar and separated) on CT, and may have a higher recurrence rate.

Review of other patient characteristics than CT findings showed a significant effect of sex on TM levels; concentrations were higher in males compared to females. ${ }^{27)}$ However, our study found neither age nor sex affected the values of any cytokines. Similar to previous findings, 29 (85.3\%) of our hematomas were males and $5(14.7 \%)$ were females. ${ }^{13)}$ Mean age (73.8 years) of our patients was also similar to other studies. ${ }^{13,17)}$

In conclusion, we found high levels of TM, IL-6, IL-10, and $\mathrm{TNF} \alpha$ in the hematoma contents of $\mathrm{CSDH}$. Evaluation of these cytokine levels according to CT appearance found only the TM values showed significant differences. However, the SD of the TM values was high, indicating wide dispersion of TM, so we will continue our investigation with more cases. Therefore, at this time, it may be more correct to say that we have trends in the high level of TM values with CT appearance rather than significant differences in TM values. Local inflammatory changes must have some effect on the pathogenesis of CSDH, but we were unable to clearly delineate the involvement of inflammatory and anti-inflammatory cytokines in this study. As reported previously, mixed density hematomas had a high recurrence rate, and TM level was high in mixed density hematomas in our study. Considering this relationship, we suggest that the types of CSDH associated with high TM values (mixed density, laminar, and separated hematomas) tend to have higher recurrence rates. The TM level can be measured objectively, in contrast to the subjective CT appearance, so may be a good index for anticipating the recurrence of CSDH. We consider that the pathogenesis is likely to involve endothelial cell injury at the outer membrane of CSDH resulting in increased TM level, with resulting higher anticoagulant function increasing the hematoma size and causing the recurrence of CSDH. Therefore, medication which inhibits TM may potentially decrease the recurrence rate of CSDH. Clinicians treating mixed density, laminar, and separated type hematomas, which may have high TM values, should be aware of the increased risk of recurrence.

\section{References}

1) Abouzari M, Rashidi A, Rezaii J, Esfandiari K, Asadollahi M, Aleali H, Abdollahzadeh M: The role of postoperative patient posture in the recurrence of traumatic chronic subdural hematoma after burrhole surgery. Neurosurgery 61: 794-797, 2007
2) Berghauser Pont LM, Dammers R, Schouten JW, Lingsma HF, Dirven CM: Clinical factors associated with outcome in chronic subdural hematoma: a retrospective cohort study of patients on preoperative corticosteroid therapy. Neurosurgery 70: 873880, 2012

3) Carlsen JG, Cortnum S, Sørensen JC: Recurrence of chronic subdural haematoma with and without postoperative drainage. Br J Neurosurg 25: 388-390, 2011

4) Delgado-López PD, Martín-Velasco V, Castilla-Díez JM, Rodríguez-Salazar A, Galacho-Harriero AM, Fernández-Arconada $\mathrm{O}$ : Dexamethasone treatment in chronic subdural haematoma. Neurocirugia (Astur) 20: 346-359, 2009

5) Dittman WA, Majerus PW: Structure and function of thrombomodulin: a natural anticoagulant. Blood 75: 329-336, 1990

6) Esmon NL: Thrombomodulin. Prog Hemost Thromb 9: 29-55, 1989

7) Feng JF, Jiang JY, Bao YH, Liang YM, Pan YH: Traumatic subdural effusion evolves into chronic subdural hematoma: Two stages of the same inflammatory reaction? Med Hypotheses 70: 1147-1149, 2008

8) Frati A, Salvati M, Mainiero F, Ippoliti F, Rocchi G, Raco A, Caroli E, Cantore G, Delfini R: Inflammation makers and risk factors for recurrence in 35 patients with a posttraumatic chronic subdural hematoma: a prospective study. J Neurosurg 100: 24-32, 2004

9) Fujioka S, Matukado Y, Kaku M, Sakurama N, Nonaka N, Miura G: [CT analysis of 100 cases with chronic subdural hematoma with respect to clinical manifestation and the enlarging process of the hematoma]. Neurol Med Chir (Tokyo) 21: 1153-1160, 1981 (Japanese)

10) Himmelreich G, Riewald M, Rosch R, Blumhard G, Neuhaus P, Roissant R, Riess H: Thrombomodulin: a marker for endothelial damage during orthotopic liver transplantation. Am J Hematol 47: 1-5, 1994

11) Hong HJ, Kim YJ, Yi HJ, Ko Y, Oh SJ, Kim JM: Role of angiogenic growth factors and inflammatory cytokine on recurrence of chronic subdural hematoma. Surg Neurol 71: 161-166, 2009

12) Ito $H$, Yamamoto $S$, Saito $K$, Ikeda $K$, Hisada $K$ : Quantitative estimation of hemorrhage in chronic subdural hematoma using the $51 \mathrm{Cr}$ erythrocyte labeling method. J Neurosurg 66: 862-864, 1987

13) Kanat A, Kayaci S, Yazar U, Kazdal H, Terzi Y: Chronic subdural hematoma in adults: why does it occur more often in males than females? Influence of patient's sexual gender on occurrence. J Neurosurg Sci 54: 99-103, 2010

14) Kishimoto T: The biology of interleukin-6. Blood 74: 1-10, 1989

15) Kuzuhara M, Nakazawa S, Matsumoto M: A study on natural history of chronic subdural hematomas by analyzing CT findings. Nihon Ika Daigaku Zasshi 51: 390-392, 1984

16) Lim DJ, Chung YG, Park YK, Song JH, Lee HK, Lee $\mathrm{KC}$, Chu JW, Yang YS: Relationship between tissue 
plasminogen activator, plasminogen activator inhibitor and CT image in chronic subdural hematoma. J Korean Med Sci 10: 373-378, 1995

17) Miranda LB, Braxton E, Hobbs J, Quigley MR: Chronic subdural hematoma in the elderly: not a benign disease. J Neurosurg 114: 72-76, 2011

18) Murakami H, Hirose $Y$, Sagoh M, Shimizu K, Kojima M, Gotoh K, Mine Y, Hayashi T, Kawase T: Why do chronic subdural hematomas continue to grow slowly and not coagulate? Role of thrombomodulin in the mechanism. J Neurosurg 96: 877-884, 2002

19) Nakaguchi H, Tanishima T, Yoshimatsu N: Factors in the natural history of chronic subdural hematomas that influence their postoperative recurrence. J Neurosurg 95: 256-262, 2001

20) Nomura S, Kashiwagi S, Fujisawa $H$, Ito $H$, Nakamura K: Characterization of local hyperfibrinolysis in chronic subdural hematomas by SDS-PAGE and immunoblot. J Neurosurg 81: 910-913, 1994

21) Owen WG, Esmon CT: Functional properties of an endothelial cell cofactor for thrombin-catalyzed activation of protein C. J Biol Chem 256: 5532-5535, 1981

22) Park SH, Kang DH, Park J, Hwang JH, Hwang SK, Sung JK, Hamm IS: Fibrinogen and D-dimer analysis of chronic subdural hematomas and computed tomography findings: A prospective study. Clin Neurol Neurosurg 113: 272-276, 2011

23) Park SH, Lee SH, Park J, Hwang JH, Hwang SK, Hamm IS: Chronic subdural hematoma preceded by traumatic subdural hygroma. J Clin Neurosci 15: 868-872, 2008

24) Patterson PH: The emerging neuropoietic cytokine family: first CDF/LIF, CNTF and IL6; next ONC, MGF, GCSF? Curr Opin Neurobiol 2: 94-97, 1992

25) Saito K, Ito H, Hasegawa T, Yamamoto S: Plasmin-alpha 2-plasmin inhibitor complex and alpha 2-plasmin inhibitor in chronic subdural hematoma. J Neurosurg 70: 68-72, 1989

26) Santarius T, Kirkpatrick PJ, Ganesan D, Chia HL, Jalloh I, Smielewski P, Richards HK, Marcus H, Parker RA, Price SJ, Kirollos RW, Pickard JD, Hutchinson PJ: Use of drains versus no drains after burr-hole evacuation of chronic subdural haematoma randomized controlled trial. Lancet 374: 1067-1073, 2009

27) Strijbos $\mathrm{MH}$, Rao C, Schmitz PI, Kraan J, Lamers CH, Sleijfer S, Terstappen LW, Gratama JW: Correlation between circulating endothelial cell counts and plasma thrombomodulin levels as markers for endothelial damage. Thromb Haemost 100: 642-647, 2008

28) Stroobandt G, Fransen P, Thauvoy C, Menard E: Pathogenetic factors in chronic subdural haematoma and causes of recurrence after drainage. Acta Neurochir (Wien) 137: 6-14, 1995

29) Suzuki M, Endo S, Inada K, Kudo A, Kitakami A,
Kuroda K, Ogawa A: Inflammatory cytokines locally elevated in chronic subdural haematoma. Acta Neurochir (Wien) 140: 51-55, 1998

30) Takahashi Y, Mikami J, Ueda M, Ito K, Sato H, Matsuoka H, Takeda S, Ohkawara S: [Analysis of chronic subdural hematoma based on CT (Part III). Clinical stage classification based on CT findings]. Neurol Med Chir (Tokyo) 24: 607-614, 1984 (Japanese)

31) Takano S, Kimura S, Ohdama S, Aoki N: Plasma thrombomodulin in health and diseases. Blood 76: 2024-2029, 1990

32) Tang J, Ai J, Macdonald RL: Developing a model of chronic subdural hematoma. Acta Neurochir Suppl 111: 25-29, 2011

33) Trotter W: Chronic subdural hemorrhage of traumatic origin, and its relation to pachymeningitis hemorrhagica interna. Br J Surg 2: 271-291, 1914

34) Tsutsumi K, Maeda K, Iijima A, Usui M, Okada Y, Kirino T: The relationship of preoperative magnetic resonance imaging findings and closed system drainage in the recurrence of chronic subdural hematoma. J Neurosurg 87: 870-875, 1997

35) Vaquero J, Zurita M, Cincu R: Vascular endothelial growth-permeability factor in granulation tissue of chronic subdural haematomas. Acta Neurochir (Wien) 144: 343-347, 2002

36) Virchow VR: [The hematoma of the dura mater]. Verh Phys Med Gesellsch Würzburg 7: 134-142, 1857 (German)

37) Wada T, Kuroda K, Yoshida Y, Ogasawara K, Ogawa A, Endo S: Local elevation of the anti-inflammatory interleukin-10 in the pathogenesis of chronic subdural hematoma. Neurosurg Rev 29: 242-245, 2006

38) Weigel R, Hohenstein A, Schlickum L, Weiss C, Schilling L: Angiotensin converting enzyme inhibition for arterial hypertension reduces the risk of recurrence in patients with chronic subdural hematoma possibly by an antiangiogenic mechanism. Neurosurgery 61: 788-792, 2007

39) Yamashita T, Yamamoto S, Friede RL: The role of endothelial gap junctions in the enlargement of chronic subdural hematomas. J Neurosurg 59: 298-303, 1983

40) Yu GJ, Han CZ, Zhang M, Zhuang HT, Jiang YG: Prolonged drainage reduces the recurrence of chronic subdural hematoma. Br J Neurosurg 23: 606-611, 2009

Address reprint requests to: Masatoshi Kitazono, MD, Department of Emergency and Critical Care Center, Kawaguchi Municipal Medical Center, 180 Nishiaraijuku, Kawaguchi, Saitama 333-0833, Japan. e-mail: s9034@nms.ac.jp 